Original article

\title{
The effect of apremilast therapy on skin cytokine levels in patients with psoriasis
}

\author{
Alexey A. Kubanov, Viktoria S. Solomka, Arfenya E. Karamova, Dmitry A. Verbenko, Elena L. Vasileva, \\ Olga G. Artamonova
}

State Research Center of Dermatovenereology and Cosmetology, Moscow, Russia

Received 11 December 2019, Revised 5 June 2020, Accepted 15 July 2020

(c) 2019, Kubanov A.A., Solomka V.S., Karamova A.E., Verbenko D.A., Vasileva E.L., Artamonova O.G.

(C) 2019, Russian Open Medical Journal

Abstract: Objective - Assessment of phosphodiesterase-4 inhibitor (apremilast) therapy's influence on skin cytokine levels in patients with moderate-to-severe and severe psoriasis.

Material and Methods - An open, uncontrolled study was conducted. 16 patients with plaque psoriasis (13 men, 3 women; mean \pm

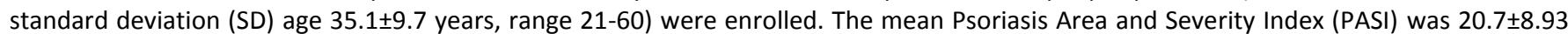
(range 10-47). All patients were prescribed apremilast 30 milligrams (mg) per os (PO) Bis In Die (BID). The efficacy of therapy was evaluated by PASI at 14 and 26 weeks of therapy. Lesional skin samples were collected at baseline and weeks 14 and 26 . Levels of interleukin (IL)-1 $\beta$, IL-4, IL-6, IL-10, IL-17A, IL-17F, IL-21, IL-22, IL-23, IL-25, IL-31, IL -33, interferon (INF)- $\gamma$, Soluble CD40-ligand (sCD40L), tumor necrosis factor (TNF)- $\alpha$ were measured by microsphere-based suspension array technology (Luminex ${ }^{\circledR}$ XMAP'M system).

Results - Levels of cytokines (except IL-4 and IL-33) in lesional skin samples were found to have decreased at week 14 compared with those at baseline. Similar decreases were seen for IL-23, IL-25, IL-31, SCD40L at week 26. In contrast, the levels of other cytokines increased again at week 26, in comparison with baseline. Levels of IL-4 and IL-33 rose throughout the follow-up period. Cytokine levels in lesional skin samples were compared with those of healthy controls both at baseline and during therapy.

Conclusion - The results of our study show that administering apremilast therapy to patients with psoriasis can bring the levels of cytokines involved in the IL-23/IL-17 axis in the lesional skin to the level of cytokine in non-lesional skin and to the levels in the skin of healthy individuals.

Keywords: psoriasis, cytokines, apremilast, phosphodiesterase-4 inhibitor.

Cite as Kubanov AA, Solomka VS, Karamova AE, Verbenko DA, Vasileva EL, Artamonova OG. The effect of apremilast therapy on skin cytokine levels in patients with psoriasis. Russian Open Medical Journal 2020; 9: e0310.

Correspondence to Olga G. Artamonova. Address: 3 bld. 6, Korolenko Street, 107076 Moscow, Russia. E-mail: artamonova_olga@list.ru.

\section{Introduction}

Plaque-type psoriasis is a chronic inflammatory skin disease involving both the innate and adaptive immune compartments, crosstalking with skin tissue cells [1]. The current pathogenetic model is based on the interleukin (IL)-23/IL-17 axis [2]. Dendritic cells influenced by multiple factors (including tumor necrosis factor (TNF)- $\alpha$ and interferon (INF)- $\gamma$ ) produce IL-23. In turn, IL-23 acts on Thelper cells (Th) 17 and Th 22, enhancing the production of IL-17 and IL-22 [2].

An example of the immune response path switching from Th1 to Th2 and vice versa is the responsibility of the pro-inflammatory cytokines IL-1 $\beta$ and IL- 6 for the Th17 differentiation [3] and the anti-inflammatory cytokine IL-4 which is produced by Th2 for the inhibition of IL-1 $\beta, \quad \mathrm{IL}-6, \mathrm{IL}-17, \mathrm{IL}-23$ expression and the differentiation of Th17 [4].

Cyclic adenosine monophosphate (cAMP) is a pivotal secondary mediator involved in regulation of inflammatory response. CAMP degradation occurs through the activity of the large superfamily of phosphodiesterases (PDE). In immune cells (including T cells, monocytes, macrophages, neutrophils, dendritic cells and eosinophils) and keratinocytes, this function is performed by phosphodiesterase (PDE)4. Inhibition of PDE4 leads to an increase in the CAMP level and inhibits the production of TNF- $\alpha$, IL12, IL-23, IL-17, IL-22 and other cytokines involved in the inflammatory reaction [5]

Apremilast is a novel PDE4 inhibitor with TNF-a, IL-10, IL-23 and IL-17A inhibitory activity [6]. The efficacy and safety of apremilast have been proven by randomized, placebo-controlled trials such as the Efficacy and Safety Trial Evaluating the Effects of Apremilast on Psoriasis (ESTEEM) 1 and 2 [7].

The goal of this study was to estimate the influence of apremilast therapy on skin cytokine levels in patients with psoriasis. The study design is based on the study of the 15 cytokine levels in the lesional skin at baseline, then at 14 and 26 weeks of therapy. Additionally, cytokine levels of non-lesional skin were compared with skin samples of the control group. 


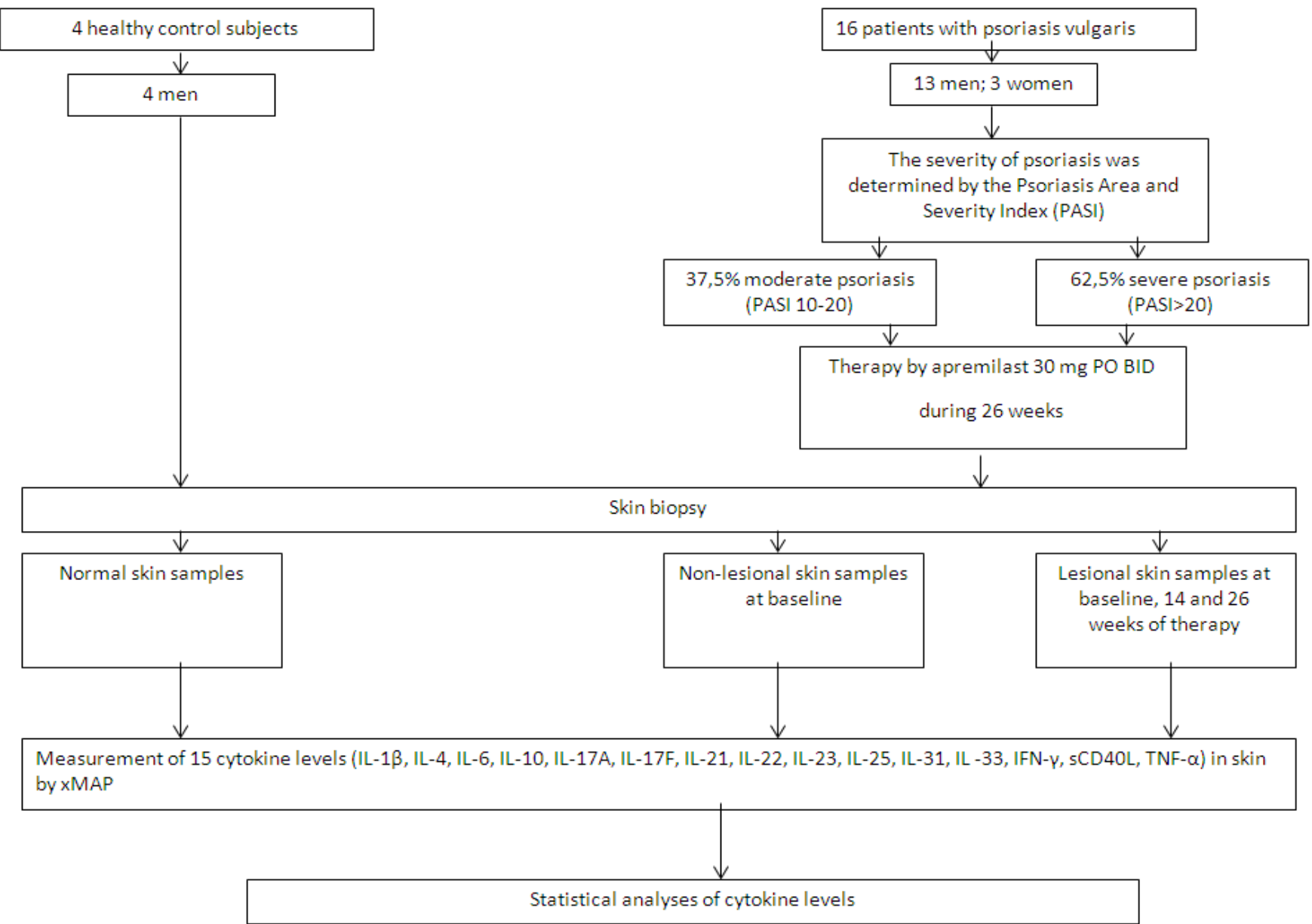

Figure 1. The study design.

Table 1. The study design

\begin{tabular}{|c|c|c|c|c|c|c|c|c|}
\hline \multirow[t]{2}{*}{ Characteristic } & \multicolumn{8}{|c|}{ Study week } \\
\hline & 0 & 2 & 6 & 10 & 14 & 18 & 22 & 26 \\
\hline Clinical blood analysis, clinical urine analysis, biochemical blood assay & + & & & & & & & + \\
\hline Blood tests for human immunodeficiency virus (HIV), hepatitis B and C, lues & + & & & & & & & \\
\hline PASI, BSA, sPGA & + & + & + & + & + & + & + & + \\
\hline DLQI & + & & & & + & & & + \\
\hline Lesional skin samples & + & & & & + & & & + \\
\hline Non-lesional skin samples & + & & & & + & & & + \\
\hline
\end{tabular}

\section{Material and Methods}

\section{Trial design}

An open, uncontrolled study was conducted. According to the study design (Figure 1), 16 patients with plaque psoriasis (13 men, 3 women; mean \pm standard deviation (SD) age 35.125 \pm 9.7 years, range 21-60) were enrolled in the study. The group's mean Psoriasis Area and Severity Index (PASI) was 20.7 \pm 8.93 (range 1047 ) and the involved body surface area (BSA) was $32.7 \pm 19.4$ (range 12-79). The severity of psoriasis was assessed as severe in 6 patients (37.5\%), with moderate severity in 10 (62.5\%). Mean duration of psoriasis was $15.75 \pm 10.27$ years (range 2-38). All patients had failed previous therapies, including phototherapy, cyclosporine, acitretin and methotrexate.

The control group comprised 4 healthy subjects (4 men, mean $\pm S D$ age $27.5 \pm 2.4$ years, range $24-29$ ).
The study was conducted in accordance with the Declaration of Helsinki's general ethical principles. Prior to the study each patient gave voluntary informed written consent, which was approved by the local ethics committee of the Federal State Research Center of Dermatovenereology and Cosmetology, Moscow, Russian Federation.

According to the research protocol, we examined the patients, recorded their body weight, collected information about adverse effects, and took pictures of the lesional skin (Table 1). Psoriasis severity was evaluated by PASI [8].

All patients were prescribed apremilast 30 milligrams $(\mathrm{mg})$ per os (PO) Bis In Die (BID). The dosing of apremilast was titrated upwards from $10 \mathrm{mg}$ PO on the first day to $30 \mathrm{mg}$ PO BID on the sixth day of treatment. The duration of therapy was 26 weeks. 

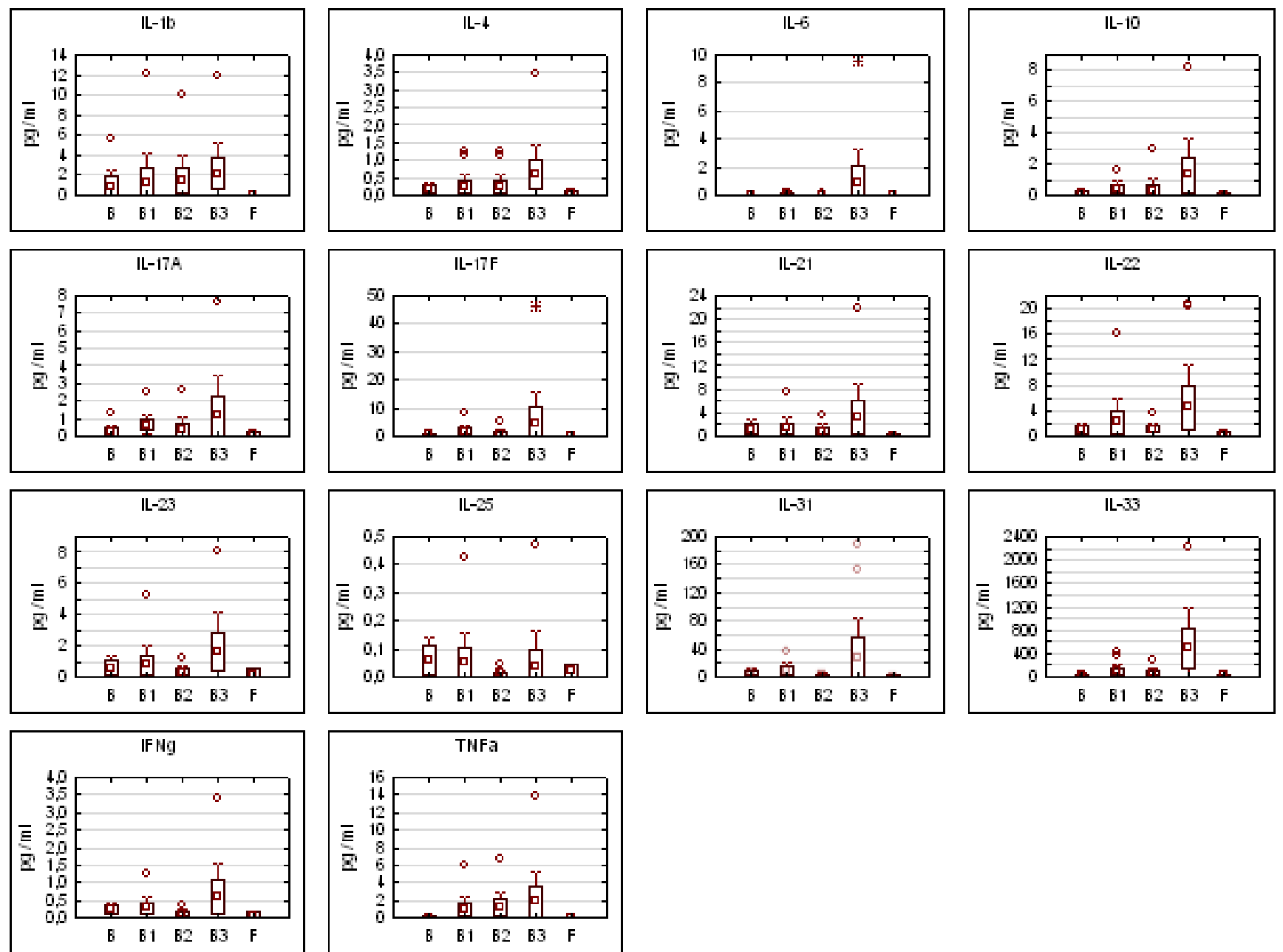

Figure 2. Cytokine levels in skin samples of patients with psoriasis and healthy subjects.

B - non-lesional skin samples of patients with psoriasis at baseline, B1 - lesional skin samples of patients with psoriasis at baseline, B2 - lesional skin samples of patients with psoriasis at week 14, B3 - lesional skin samples of patients with psoriasis at week $26, \mathrm{~F}-$ skin samples of healthy subjects.

The efficacy of therapy was evaluated by PASI, BSA, static Physician's Global Assessment (sPGA) and Dermatology Life Quality Index (DLQI) at weeks 14 and 26 of therapy (Table 1).

Lesional skin samples were collected from patients at baseline and weeks 14 and 26. Non-lesional skin samples were collected from patients only at baseline (Table 1).

\section{Determination of cytokine levels}

Skin biopsy samples (size $0.8 \times 0.5 \mathrm{~cm}$ ) were obtained from patients with psoriasis and healthy individuals. Samples were weighed on a laboratory balance in labeled 1.5-millilitre $(\mathrm{ml})$ tubes of the Eppendorf type (Axygen). Skin biopsy samples were regrounded using the automated tissue disaggregation system 'Medimachine' (BectonDickinson, USA) in special disposable chambers 'Medicons' (BectonDickinson, USA) for 2 minutes with the addition of $1 \mathrm{ml}$ of phosphate buffer $(\mathrm{pH}=7.4)$ to each sample. Before the study, the suspension was passed through a disposable filter "Filcons» (BectonDickinson, USA) with a 50 micron $(\mu \mathrm{m})$ pore size.

The level of cytokines was determined on the 'BioPlex 200' device (Bio-Rad, USA) using the commercial reagent kit 'Bio-Plex
Pro Human Th17 Cytokine Panel 15-Plex' (Bio-Rad, USA), which allowed simultaneous determination of levels of the following cytokines: IL-1 $\beta$, IL-4, IL-6, IL-10, IL-17A, IL-17F, IL-21, IL-22, IL-23, IL-25, IL-31, IL-33, IFN- $\gamma$, SCD40L and TNF- $\alpha$, according to the previously described methodology [9]. The obtained values were recalculated in accordance with the initial weight of the samples according to the formula:

Amount of cytokine in $1 \mathrm{ml}$ of skin biopsy sample in picograms (pg) / Weight of skin biopsy sample ( $\mathrm{mg})=$ Cytokine concentration in skin biopsy sample $(\mathrm{pg} / \mathrm{mg})$

\section{Statistical analysis}

Statistical data processing was carried out using Statistica13 software. The Shapiro-Wilk test was used to check the normality of the data distributions, and the Mann-Whitney test was carried out to assess the significance of differences between the groups. The levels of cytokines were indicated as the median [quartile 1; quartile 3], because the distribution of obtained values was defined as non-normal [10]. The confidence interval was set to 0.95 . 


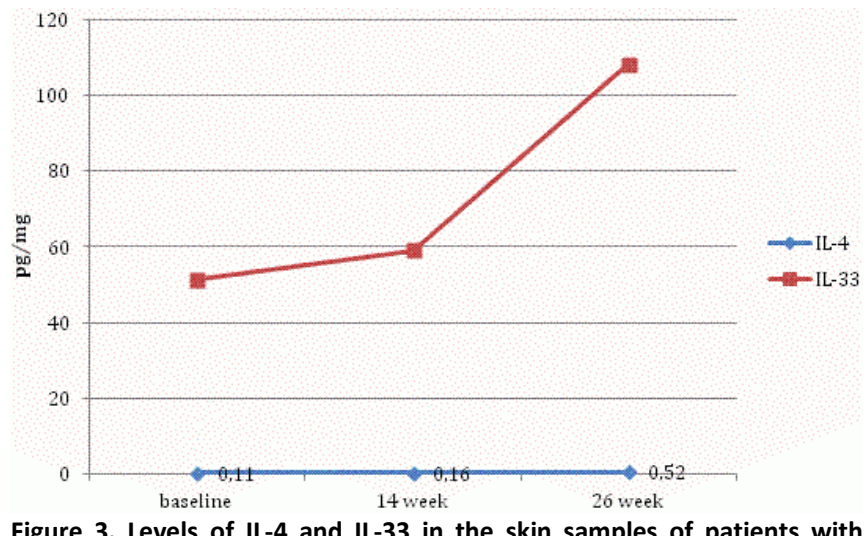

Figure 3. Levels of IL-4 and IL-33 in the skin samples of patients with psoriasis at baseline, 14 and 26 weeks of therapy.

\section{Results}

At week 14, reduction of PASI by half ( $50 \%)$ was registered in 5 patients $(31.25 \%)$, with a $75 \%$ reduction in 6 patients $(37.5 \%)$, while 5 patients $(31.25 \%)$ had not reached PASI 50 . By week 26 , reduction of PASI by half $(50 \%)$ was registered in 4 patients $(25 \%)$, with a $75 \%$ reduction in 8 patients $(50 \%)$, and 4 patients $(25 \%)$ had not reached PASI 50.

\section{Changes in cytokine levels}

In the non-lesional skin samples of patients with psoriasis $(n=10)$, at baseline the highest values of cytokine levels were detected for IL-33, IL-31, and IL-21, while the lowest values were determined for TNF-a, IL-25, and IL-6 (Table 2). The highest values of cytokine levels in the lesional skin samples of patients with psoriasis at baseline were for IL-33 and IL-31, while the lowest values were for IL-25, IL-6, INF- $\gamma$, and IL-4.

By week 14 of therapy, the highest values of cytokine levels in lesional skin samples were still for IL-33 and IL-31. The lowest values of cytokine levels in lesional skin samples were registered for IL-25, IL-6, INF- $\psi$, and IL-4. By week 26, the highest values were registered for IL-33 and IL-22, with the lowest - for IL-25, IL-6, IL31 and SCD40L (Figure 2).

Progressive increases in IL-4 and IL-33 levels were observed throughout the study (Figure 3 ).

Levels of 9 out of 15 cytokines (IL-13, IL-6, IL-10, IL-17A, IL-17F, IL-21, IL-22, IFN- $\gamma$ and TNF- $\alpha$ ) decreased by week 14 but then significantly increased by week 26 during therapy (Figure 4), irrespective of baseline level.

In addition, the largest increases during therapy in comparison with baseline data were recorded for IL-33, IL-1 $\beta$, and IL-4. Levels of IL-23, IL-25, IL-31 and SCD40L decreased throughout the study (Figure 5).

\section{Statistically significant differences between groups}

Comparison of cytokine levels in lesional skin samples obtained at baseline with cytokine levels in lesional skin samples obtained at week 14 of therapy found statistically significant differences for IL-6, IL-17A, IL-17F, IL-31 and IFNY. At the same time, no statistically significant differences were found between levels of other cytokines in lesional skin samples obtained at week 14 and those obtained at week 26. This was also the case for levels of other cytokines in the lesional skin samples obtained at baseline, and in those obtained at week 26 .

Statistically significant differences for IL-1 $\beta$, IL-6, IL-17A, IL-17F and TNF $\alpha$ levels were found in non-lesional skin samples in comparison with the lesional skin samples obtained at baseline.

Comparison of cytokine levels in skin samples the control group with non-lesional skin samples from patients with psoriasis revealed no statistically significant differences, indicating the absence of noticeable disturbances in the intercellular signaling system in the non-lesional skin of patients with psoriasis. Comparison of cytokine levels in lesional skin samples obtained at baseline with skin samples from the control group revealed a greater number of statistically significant differences for IL-21, IL22, IL-31, IL-1ß, IL-6, IL-17A, IL-17F and TNF $\alpha$ levels. Analysis of the control group data also revealed statistically significant differences for TNF $\alpha$ and IL-1 $\beta$ by the week 14 of therapy, and for IL-22, IL-33, IL-1 $\beta$ and TNF $\alpha$ - by week 26 .

Table 2. The highest and lowest values of cytokine levels in skin samples of patients with psoriasis

\begin{tabular}{|c|c|c|c|c|}
\hline Skin biopsy & The $h$ & levels, $p g / m g$ & The low & els, $p g / m g$ \\
\hline & IL-33 & $22,30[22,30 ; 51,67]$ & TNF-a & $0,06[0,06 ; 0,14]$ \\
\hline Non-lesional skin samples, & IL-31 & $3,63[3,63 ; 7,98]$ & $\mathrm{IL}-25$ & $0,01[0,01 ; 0,1]$ \\
\hline & IL-21 & $0,48[0,48 ; 2,1]$ & IL-6 & $0[0 ; 0,01]$ \\
\hline & IL-33 & $51,45[32,08 ; 86,34]$ & $\mathrm{IL}-25$ & $0,03[0 ; 0,05]$ \\
\hline Lesional skin samples, & IL-31 & $4,87[3,32 ; 7,16]$ & IL-6 & $0,07[0 ; 0,23]$ \\
\hline baseline & & & INF- $\gamma$ & $0,17[0,14 ; 0,21]$ \\
\hline & & & $\mathrm{IL}-4$ & $0,11[0 ; 0,26]$ \\
\hline & IL-33 & $59,15[25,79 ; 143,24]$ & $\mathrm{IL}-25$ & $0[0 ; 0,02]$ \\
\hline Lesional skin samples, & IL-31 & $3,27[0 ; 4,84]$ & IL-6 & $0[0 ; 0,01]$ \\
\hline Week 14 & & & INF- $\psi$ & $0,08[0,01 ; 0,16]$ \\
\hline & & & IL-4 & $0,16[0 ; 0,40]$ \\
\hline & IL-33 & $108,25[41,78 ; 646,35]$ & $\mathrm{IL}-25$ & $0[0 ; 0]$ \\
\hline Lesional skin samples, & $\mathrm{IL}-22$ & $1,43[0,69 ; 5,89]$ & IL-6 & $0,01[0 ; 0,03]$ \\
\hline Week 26 & & & IL-31 & $0[0 ; 8,89]$ \\
\hline & & & SCD40L & $0,01[0 ; 0,93]$ \\
\hline
\end{tabular}


Table 3. The biological role of cytokines in psoriasis [14]

\begin{tabular}{|c|c|c|}
\hline Cytokine Family & Cytokine functions & Cytokine type \\
\hline Interferons (IFN) & $\begin{array}{l}\text { Antiviral activity, antiproliferative, immunomodulatory effect. } \\
\text { In psoriasis, IFN- } \gamma \text { decreases the activity of T-helpers } 2 \text { and increases the activity of T-killers. }\end{array}$ & IFN- $\alpha$, IFN- $\beta$, IFN- $\gamma$ \\
\hline Tumor necrosis factor (TNF) & Pro-inflammatory effect, regulation of apoptosis and intercellular interaction of immune cells. & TNF- $\alpha$, TNF- $\beta$ \\
\hline Interleukine (IL)-1 family & Pro-inflammatory effect, activation of specific immunity, initiation of TNF production in psoriasis. & $\mathrm{IL}-1 \alpha, \mathrm{IL}-1 \beta$ \\
\hline Interleukine (IL)-6 family & Immunoregulatory effect. & IL-6, IL-11, IL-31 \\
\hline Interleukine (IL)-10 family & Immunosuppressive effect. & IL-10, IL-19, IL-20, IL-22, IL-24, IL-26 \\
\hline Interleukine (IL)-10 family & Regulation of T-lymphocytes differentiation & $\mathrm{IL}-12, \mathrm{IL}-23, \mathrm{IL}-27$ \\
\hline T-helper cytokines & Activation of humoral immunity & $\begin{array}{c}\text { T-helpers 1: } \\
\text { IL-2, IL-15, IL-21, TNF, IFN- } \gamma \\
\text { T-helpers 2: } \\
\text { IL-4, IL-5, IL-10, IL-13, IL-25 }\end{array}$ \\
\hline Interleukine (IL)-17 family & Activation of the pro-inflammatory cytokines synthesis & IL-17A, B, C, D, E, F \\
\hline
\end{tabular}

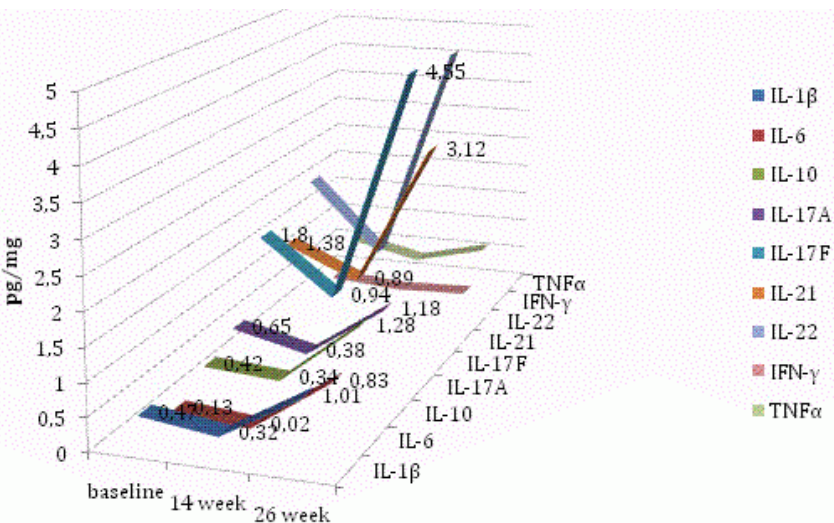

Figure 4. Levels of IL-1 $\beta$, IL-6, IL-10, IL-17A, IL-17F, IL-21, IL-22, IFN- $\gamma$ and TNF- $\alpha$ in the skin samples of patients with psoriasis at baseline, 14 and 26 weeks of therapy.

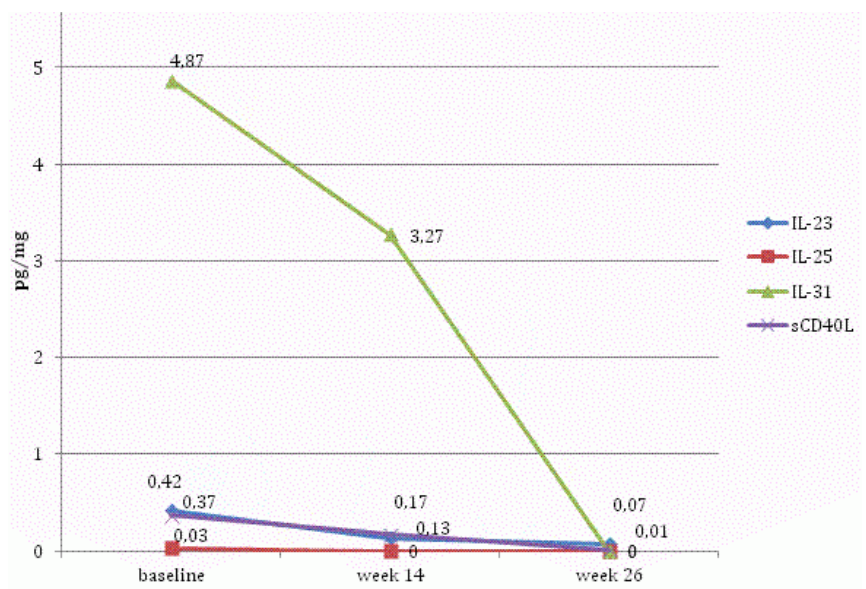

Figure 5. Levels of IL-23, IL-25, IL-31 and SCD40L in the skin samples of patients with psoriasis at baseline, 14 and 26 weeks of therapy.

\section{Discussion}

There are many studies of blood cytokine levels in patients with psoriasis [11-13], but less is known about cytokine levels in the skin. Abdel-Hamid MF et al. (2011) showed that serum levels of TNF- $\alpha$ and IFN- $\gamma$ in patients with psoriasis are statistically significantly higher than in healthy individuals [11]. Hwang et al. (2014) noted elevated serum levels of IFN- $\gamma$, IL-1RA, IL-2 and IL-23 [12]. Cataldi et al. (2019) recorded increased serum levels of IL-1 $\beta$, TNF- $\alpha$ of IL- 6 and IL-17 in patients with psoriasis in comparison with healthy individuals [14].
In our study, for the first time, skin cytokine levels of a control group were analyzed and compared with samples of lesional and non-lesional skin from patients with psoriasis. Additionally, we studied the effect of PDE-4 inhibitor therapy on skin cytokine levels in patients with psoriasis. The levels of cytokines in the lesional skin were measured at baseline, as well as at week 14 (second stage) and week 26 (third stage) of the therapy.

It was found that the cytokine levels in non-lesional skin approached the skin cytokine levels of healthy individuals, while in lesional skin. there were increases in the cytokine levels of IL-1 $\beta$, IL-6, IL-17A, IL-17F, IL -22, IL-31 and TNF $\alpha$. This finding is consistent with the biological role of these mediators in psoriasis as proinflammatory and immunoregulatory agents (Table 3 ). The data obtained are consistent with the results of the ESTEEM 2 and PSOR-011 (prior dose-finding study of apremilast in adults with moderate-to-severe psoriasis) trials $[15,16]$, where blood levels of IL-17A, IL-17F and IL-22 were found to be higher in patients with psriasis than in healthy individuals. A decrease in the levels of 13 cytokines level (IL-1 $\beta$ and IL-33 levels were the exceptions) was observed in the lesional skin by week 14 of the PDE-4 inhibitor therapy. Decreases in levels of IL-23, IL-25, IL-31 and SCD40L levels were sustained to week 26 of therapy. In contrast, levels of the other cytokines studied increased by week 26 , exceeding the baseline level.

The results of statistical analysis showed statistically significant decreases for IL-1 $\beta$, IL-6, IL17A, IL17F and TNF $\alpha$ levels by week 14 of therapy and no statistically significant increase of any of the 15 cytokine levels by week 26 of therapy. Despite the fact that increased levels of IL-4 and IL-33 were observed in lesional skin in week 14, no statistically significant differences in the levels of these cytokines were found either between the first and second, or second and third stages of the study.

It was observed that IL-1 $\beta$, IL-6, IL-10, IL-17A, IL-17F, IL-21, IL22, IFN- $\gamma$ and TNF- $\alpha$ levels had all decreased by week 14 of therapy, but had increased again by week 26 . It should be noted that the increase in these cytokine levels did not lead to a deterioration of patients' psoriasis.

Garcet et al. (2018) also noted decreases in blood levels of IL17A, IL-17F, IL-22 and TNF- $\alpha$ in patients with psoriasis by 4 and 16 weeks of apremilast therapy, which was accompanied by clinical improvement [17].

Comparison of levels of the 15 cytokines in lesional skin during therapy with the baseline level of cytokines in non-lesional skin in the same patients showed: statistically significant differences for 5 cytokines (IL-1 $\beta$, IL-6, IL-17A, IL- 17F and TNF $\alpha$ ) at baseline, for 1 
cytokine (TNF- $\alpha$ ) at week 14 , for 2 cytokines (TNF- $\alpha$ and IL-31) at week 26.

Comparison of levels of the 15 cytokines in lesional skin samples obtained at weeks 14 and 26 with cytokine levels in skin samples from healthy individuals showed the same positive changes in the processes of intercellular signaling during therapy. At baseline, statistically significant differences were observed for 8 out of 15 cytokines, by the week 14 for only 2 cytokines, and by week 26 for 4 .

Thus, the complex mechanism for the transmission of intercellular signals, involving the interaction of skin cells and immune cells, with the IL-23/IL-17 axis at its centre, is significantly impaired in the lesional skin of patients with psoriasis where is an accompanying increase in pro-inflammatory cytokine levels. Under the apremilast therapy in the study, these levels approached the level of cytokines in non-lesional skin and those in the skin of healthy individuals.

\section{Conclusion}

The results of our study show that administering apremilast therapy to patients with psoriasis can bring the levels of cytokines involved in the IL-23/IL-17 axis in the lesional skin to the level of cytokine in non-lesional skin and to the levels in the skin of healthy individuals.

\section{Conflict of interest}

The authors declare that they have no conflict of interest.

\section{Funding}

This study was funded by grant No18-15-00372 of the Russian Science Foundation ('The prediction of a response to targeted therapy of severe psoriasis').

\section{Ethical approval}

All procedures performed in studies involving human participants were in accordance with the ethical standards of the institutional and/or national research committee and with the 1964 Helsinki declaration and its later amendments or comparable ethical standards.

\section{References}

1. Chiricozzi A, Romanelli P, Borsellino G, Volpe E, Romanelli M. Scanning the immunopathogenesis of psoriasis. Int J Mol Sci 2018; 19(1): 179. https://doi.org/10.3390/ijms19010179.

2. Gisondi P, Bellinato F, Girolomoni G, Albanesi C. Pathogenesis of Chronic Plaque Psoriasis and Its Intersection With Cardio-Metabolic Comorbidities. Front Pharmacol 2020; 11: 117. https://doi.org/10.3389/fphar.2020.00117.

3. Dowlatshahi EA, van der Voort EA, Arends LR, Nijsten T. Markers of systemic inflammation in psoriasis: a systematic review and metaanalysis. $\mathrm{Br} J$ Dermatol 2013; 169(2): 266-282. https://doi.org/10.1111/bjd.12355

4. Hahn M, Ghoreschi K. The role of IL-4 in psoriasis. Expert Rev Clin Immunol 2017; 13(3): 171-173. https://doi.org/10.1080/1744666x.2017.1279054.

5. Schafer PH, Parton A, Gandhi AK, Capone L, Adams M, Wu L, et al. Apremilast, a cAMP phosphodiesterase-4 inhibitor, demonstrates antiinflammatory activity in vitro and in a model of psoriasis. $\mathrm{Br} J$ Pharmacol 2010; 159(4): 842-855. https://doi.org/10.1111/j.14765381.2009.00559.x
6. Vujic I, Herman R, Sanlorenzo M, Posch C, Monshi B, Rappersberger K, et al. Apremilast in psoriasis - a prospective real-world study. J Eur Acad Dermatol Venereol 2018; 32(2):254-259. https://doi.org/10.1111/jdv.14598.

7. Papp K, Reich K, Leonardi C, Kircik L, Chimenti S, Langley RG, et al. Apremilast, an oral phosphodiesterase 4 (PDE4) inhibitor, in patients with moderate to severe plaque psoriasis: Results of a phase III, randomized, controlled trial (Efficacy and Safety Trial Evaluating the Effects of Apremilast in Psoriasis [ESTEEM] 1). J Am Acad Dermatol 2015; 73(1): 37-49. https://doi.org/10.1016/j.jaad.2015.03.049.

8. Puzenat E, Bronsard V, Prey S, Gourraud PA, Aractingi S, Bagot M, et al. What are the best outcome measures for assessing plaque psoriasis severity? A systematic review of the literature. J Eur Acad Dermato Venereol 2010; 24 Suppl 2; 10-16. https://doi.org/10.1111/i.14683083.2009.03562.x.

9. Hall SA, Stucke D, Morrone B, Lebelt D, Zanella AJ. Simultaneous detection and quantification of six equine cytokines in plasma using a fluorescent microsphere immunoassay (FMIA). Methods. 2015; 2: 241 248. https://doi.org/10.1016/i.mex.2015.04.002.

10. Lang A.T., Altman D.G. Basic statistical reporting for articles published in biomedical journals: the "statistical analyses and methods in the published literature" or the SAMPL guidelines. Int J Nurs Stud 2015; 52(1): 5-9. https://doi.org/10.1016/j.ijnurstu.2014.09.006.

11. Abdel-Hamid MF, Aly DG, Saad NE, Emam HM, Ayoub DF. Serum levels of interleukin-8, tumor necrosis factor- $\alpha$ and $\gamma$-interferon in Egyptian psoriatic patients and correlation with disease severity. I Dermatol 2011; 38(5): 442-446. $\quad$ https://doi.org/10.1111/i.13468138.2010.01018.x.

12. Hwang YJ, Jung HJ, Kim MJ, Roh NK, Jung JW, Lee YW et al. Serum levels of LL-37 and inflammatory cytokines in plaque and guttate psoriasis. Mediators Inflamm 2014; 2014: 268257. https://doi.org/10.1155/2014/268257.

13. Cataldi C., Mari NL, Lozovoy MAB, Martins LMM, Reiche EMV, Maes M et al. Proinflammatory and anti-inflammatory cytokine profiles in psoriasis: use as laboratory biomarkers and disease predictors. Inflamm Res 2019; 68(7): 557-567. https://doi.org/10.1007/s00011 019-01238-8.

14. Simbirtsev AS. Cytokines - classification and biologic functions. Cytokines and Inflammation 2004; 3(2): 16-22. Russian. https://elibrary.ru/item.asp?id=9124580.

15. Paul C, Cather J, Gooderham M, Poulin Y, Mrowietz U, Ferrandiz C, et al. Efficacy and safety of apremilast, an oral phosphodiesterase 4 inhibitor, in patients with moderate-to-severe plaque psoriasis over 52 weeks: a phase III, randomized controlled trial (ESTEEM 2). $\mathrm{Br} J$ Dermatol 2015; 173(6): 1387-1399. https://doi.org/10.1111/bjd.14164.

16. Ohtsuki $M$, Okubo $Y$, Komine $M$, Imafuku S, Day RM, Chen $P$, et al. Apremilast, an oral phosphodiesterase 4 inhibitor, in the treatment of Japanese patients with moderate to severe plaque psoriasis: Efficacy, safety and tolerability results from a phase $2 \mathrm{~b}$ randomized controlled trial. J Dermatol 2017; 44(8): 873-884. https://doi.org/10.1111/13468138.13829.

17. Garcet S, Nograles K, Correa da Rosa J, Schafer PH, Krueger JG. Synergistic cytokine effects as apremilast response predictors in patients with psoriasis. J Allergy Clin Immunol 2018; 142(3): 10101013.e6. https://doi.org/10.1016/j.jaci.2018.05.039.

\section{Authors:}

Alexey A. Kubanov - MD, PhD, Director of State Research Center of Dermatovenereology and Cosmetology, State Research Center of Dermatovenereology and Cosmetology, Moscow, Russia. http://orcid.org/0000-0002-7625-0503.

Viktoria S. Solomka - MD, PhD, deputy director for Research, State Research Center of Dermatovenereology and Cosmetology, Moscow, Russia. https://orcid.org/0000-0002-6841-8599. 
Arfenya E. Karamova - MD, PhD, Head of Dermatology Department, State Research Center of Dermatovenereology and Cosmetology, Moscow, Russia. http://orcid.org/0000-0003-3805-8489.

Dmitry A. Verbenko - MD, PhD, Head of Laboratory Diagnosis of STD and Dermatoses Department, State Research Center of Dermatovenereology and Cosmetology, Moscow, Russia. https://orcid.org/0000-0002-11047694.

Elena L. Vasileva - MD, junior researcher of Department of Laboratory Diagnosis of STD and Dermatoses, State Research Center of Dermatovenereology and Cosmetology, Moscow, Russia. https://orcid.org/0000-0002-3580-8398.

Olga G. Artamonova - MD, PhD student, State Research Center of Dermatovenereology and Cosmetology, Moscow, Russia. https://orcid.org/0000-0003-3778-4745. 